Louisiana State University

LSU Digital Commons

Faculty Publications

Department of Physics \& Astronomy

$4-10-2011$

\title{
Photometric and spectroscopic evolution of the IIP SN 2007it to day 944
}

J. E. Andrews

Louisiana State University

B. E.K. Sugerman

Goucher College

Geoffrey C. Clayton

Louisiana State University

J. S. Gallagher

Raymond Walters College

M. J. Barlow

University College London

See next page for additional authors

Follow this and additional works at: https://digitalcommons.Isu.edu/physics_astronomy_pubs

\section{Recommended Citation}

Andrews, J., Sugerman, B., Clayton, G., Gallagher, J., Barlow, M., Clem, J., Ercolano, B., Fabbri, J., Meixner, M., Otsuka, M., Welch, D., \& Wesson, R. (2011). Photometric and spectroscopic evolution of the IIP SN 2007it to day 944. Astrophysical Journal, 731 (1) https://doi.org/10.1088/0004-637X/731/1/47

This Article is brought to you for free and open access by the Department of Physics \& Astronomy at LSU Digital Commons. It has been accepted for inclusion in Faculty Publications by an authorized administrator of LSU Digital Commons. For more information, please contact ir@lsu.edu. 


\section{Authors}

J. E. Andrews, B. E.K. Sugerman, Geoffrey C. Clayton, J. S. Gallagher, M. J. Barlow, J. Clem, B. Ercolano, J. Fabbri, M. Meixner, M. Otsuka, D. L. Welch, and R. Wesson 


\title{
PHOTOMETRIC AND SPECTROSCOPIC EVOLUTION OF THE IIP SN 2007it TO DAY 944
}

\author{
J. E. Andrews ${ }^{1}$, B. E. K. Sugerman ${ }^{2}$, Geoffrey C. Clayton ${ }^{1}$, J. S. Gallagher ${ }^{3}$, M. J. Barlow ${ }^{4}$, J. Clem $^{1}$, B. Ercolano ${ }^{5}$,

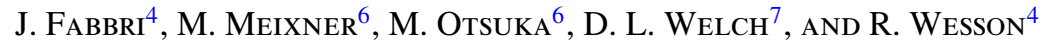 \\ ${ }^{1}$ Department of Physics and Astronomy, Louisiana State University, 202 Nicholson Hall, Baton Rouge, LA 70803, USA; jandrews@ phys.1su.edu, \\ jgallagher@phys.1su.edu, gclayton@fenway.phys.1su.edu, jclem@phys.lsu.edu \\ ${ }^{2}$ Department of Physics and Astronomy, Goucher College, 1021 Dulaney Valley Rd., Baltimore, MD 21204, USA; ben.sugerman@goucher.edu \\ ${ }^{3}$ Department of Mathematics, Physics, and Computer Science, Raymond Walters College, 9555 Plainfield Rd., Blue Ash, OH 45236, USA; gallagj1@ ucmail.uc.edu \\ ${ }^{4}$ Department of Physics and Astronomy, University College London, Gower Street, London WC1E 6BT, UK; mjb@ star.ucl.ac.uk, jfabbri@ star.ucl.ac.uk, \\ rwesson@star.ucl.ac.uk \\ ${ }^{5}$ Astrophysics Group, University of Exeter, Stocker Road, Exeter, EX4 4QL, UK; barbara@ astro.ex.ac.uk \\ ${ }^{6}$ Space Telescope Science Institute, 3700 San Martin Drive, Baltimore, MD 21218, USA; meixner@ stsci.edu, otsuka@ stsci.edu \\ ${ }^{7}$ Department of Physics and Astronomy, McMaster University, Hamilton, Ontario L8S 4M1, Canada; welch@ physics.mcmaster.ca \\ Received 2010 September 28; accepted 2011 February 11; published 2011 March 22
}

\begin{abstract}
SN 2007it is a bright, Type IIP supernova which shows indications of both pre-existing and newly formed dust. The visible photometry shows a bright late-time luminosity, powered by the $0.09 M_{\odot}$ of ${ }^{56} \mathrm{Ni}$ present in the ejecta. There is also a sudden drop in optical brightness after day 339, and a corresponding brightening in the IR due to new dust forming in the ejecta. $\mathrm{CO}$ and $\mathrm{SiO}$ emission, generally thought to be precursors to dust formation, may have been detected in the mid-IR photometry of SN 2007it. The optical spectra show stronger than average [O I] emission lines and weaker than average [Ca II] lines, which may indicate a 16-27 $M_{\odot}$ progenitor, on the higher end of expected Type IIP masses. Multi-component [O I] lines are also seen in the optical spectra, most likely caused by an asymmetric blob or a torus of oxygen core material being ejected during the $\mathrm{SN}$ explosion. Interaction with circumstellar material prior to day 540 may have created a cool dense shell between the forward and reverse shocks where new dust is condensing. At late times there is also a flattening of the visible light curve as the ejecta luminosity fades and a surrounding light echo becomes visible. Radiative transfer models of SN 2007it spectral energy distributions indicate that up to $10^{-4} M_{\odot}$ of new dust has formed in the ejecta, which is consistent with the amount of dust formed in other core-collapse supernovae.
\end{abstract}

Key words: circumstellar matter - dust, extinction - supernovae: general - supernovae: individual (SN 2007it)

Online-only material: color figures

\section{INTRODUCTION}

Type II supernovae ( $\mathrm{SNe}$ ) are core-collapse supernovae $(\mathrm{CCSNe})$ whose spectra show the presence of hydrogen. They are sub-divided based on light-curve evolution, and Type IIP $\mathrm{SNe}$ show an extended plateau phase lasting $\sim 70-110$ days due to radiative cooling of the shock-heated hydrogen envelope as well as energy released from hydrogen recombination (Grassberg et al. 1971). This phase is then followed by a tail of linear decline in luminosity, due to the radioactive decay of ${ }^{56} \mathrm{Co}$, with the luminosity being constrained by the amount of ejected ${ }^{56} \mathrm{Ni}$ (Weaver \& Woosley 1980). Type IIP SNe make up almost $60 \%$ of CCSNe (Smartt et al. 2009). The progenitors of this subclass are thought to be red supergiants (RSG) with masses between 8 and $25 M_{\odot}$ (Eldridge \& Tout 2004; Heger et al. 2003) that steadily lose their atmospheres with winds of $10-15 \mathrm{~km} \mathrm{~s}^{-1}$. Recently, a comprehensive analysis by Smartt et al. (2009) did not find any evidence for progenitors above $17 M_{\odot}$, which may indicate a disconnect between theory and observation since theoretical models of CCSNe progenitors, such as those listed in Maguire et al. (2010), have estimated progenitor masses of up to $29 M_{\odot}$. Therefore, the search is ongoing for detection of more massive progenitors.

The study of the role of CCSNe as major contributors to dust production in the universe has grown dramatically over the last few years. This is due to the discovery of high-redshift galaxies containing massive amounts of dust (Bertoldi et al. 2003). The origin of this dust is still under much contention, whether it be from quick, explosive processes such as from
CCSNe or from slow, quiescent processes such as from the atmospheres of asymptotic giant branch (AGB) stars. CCSNe, which can quickly return their material back into the surrounding interstellar medium (ISM), are attractive potential producers of dust seen at high- $z$. Up to $1 M_{\odot}$ of dust per SN would be needed to account for the dust seen at high- $z$ according to the work of Todini \& Ferrara (2001) and Nozawa et al. (2003). Numerous comprehensive studies of nearby $\mathrm{SNe}$ have been undertaken in order to estimate dust masses, but at the most only $10^{-2} M_{\odot}$ of newly formed dust has been detected (Sugerman et al. 2006), with the average newly formed dust masses being $10^{-3}-10^{-4} M_{\odot}$ (Elmhamdi et al. 2003; Meikle et al. 2007; Kotak et al. 2009, for example). Low-mass stars take much longer to evolve into the dust producing AGB phase, and may not have had enough time to evolve to become major dust producers in galaxies less than $1 \mathrm{Gyr}$ old as they do in modern galaxies (Dwek et al. 2007; Michałowski et al. 2010). Although, recent papers by Valiante et al. (2009), Sloan et al. (2009), and Dwek \& Cherchneff (2011) suggest AGB stars may be significant dust contributors as early as $\sim 150-500 \mathrm{Myr}$. Michałowski et al. (2010) find that in six submillimeter galaxies with $z>4$, the dust content of three can be explained by AGB stars, while the remaining three can only be explained by CCSNe.

Dust formation in the ejecta of CCSNe can be detected by certain observational signatures. These include a decrease in the optical luminosity due to dust grains absorbing the visible light, while at the same time creating an excess in the IR as the grains re-emit the light at longer wavelengths. Lastly, the spectral lines may appear to be asymmetric and blueshifted as 
Table 1

Photometry Summary

\begin{tabular}{lccccc}
\hline \hline Day & JD & Telescope & Instrument & Exposures & Exposure Time (s) \\
\hline 107 & 2454455 & SMARTS $1.3 \mathrm{~m}$ & ANDICAM & 5 & 20 \\
113 & 2454461 & SMARTS $1.3 \mathrm{~m}$ & ANDICAM & 5 & 20 \\
139 & 2454487 & SMARTS $1.3 \mathrm{~m}$ & ANDICAM & 5 & 20 \\
157 & 2454505 & SMARTS $1.3 \mathrm{~m}$ & ANDICAM & 5 & 20 \\
188 & 2454536 & SMARTS $1.3 \mathrm{~m}$ & ANDICAM & 5 & 20 \\
209 & 2454557 & Gemini South & GMOS Imaging & 3 & 20 \\
223 & 2454571 & SMARTS $1.3 \mathrm{~m}$ & ANDICAM & 5 & 20 \\
242 & 2454590 & Gemini South & GMOS Imaging & 3 & 20 \\
252 & 2454600 & SMARTS 1.3m & ANDICAM & 3 & 100 \\
276 & 2454624 & SMARTS 1.3m & ANDICAM & 3 & 100 \\
279 & 2454627 & Gemini South & GMOS Imaging & 3 & 20 \\
300 & 2454648 & SMARTS $1.3 \mathrm{~m}$ & ANDICAM & 3 & 100 \\
302 & 2454650 & Gemini South & GMOS Imaging & 3 & 20 \\
314 & 2454662 & SMARTS 1.3m & ANDICAM & 3 & 100 \\
339 & 2454687 & Gemini South & GMOS Imaging & 3 & 20 \\
351 & 2454699 & Spitzer & IRAC & 12 & 100 \\
540 & 2454888 & Gemini South & GMOS Imaging & 1 & 60 \\
561 & 2454909 & Spitzer & IRAC & 12 & 100 \\
566 & 2454914 & Gemini South & GMOS Imaging & 2 & 60 \\
589 & 2454937 & Gemini South & GMOS Imaging & 2 & 60 \\
613 & 2454961 & HST & WFPC2 & 4 & 412 \\
622 & 2454970 & Gemini South & GMOS Imaging & 2 & 60 \\
696 & 2455044 & HST & ACS/WFC & 4 & 412 \\
718 & 2455066 & Spitzer & IRAC & 12 & 60 \\
916 & 2455264 & Gemini South & GMOS Imaging & 2 & 424 \\
922 & 2455270 & HST & ACS/WFC & 4 & \\
& & & & & \\
\hline
\end{tabular}

the dust grains obscure the receding (red) side of the ejecta more so than the approaching (blue) side. All three of these signatures were seen for the first time in SN 1987A (Lucy et al. 1989; Wooden et al. 1993), then in SN 2003gd (Sugerman et al. 2006; Meikle et al. 2007), and also SN 2004et (Sahu et al. 2006; Kotak et al. 2009). There have also been several CCSNe in the past few years that have shown one or two of these indicators of dust formation (Kozasa et al. 2009, and references therein). Although these signatures usually appear between 1 and 2 years after the explosion, there has recently been confirmation of dust forming much earlier (less than 200 days) in a few CCSNe with circumstellar interaction. Normal Type II RSG progenitors only lose about $10^{-6}-10^{-5} M_{\odot} \mathrm{yr}^{-1}$ (Chevalier et al. 2006), which is too tenuous to show any circumstellar medium (CSM) interaction. The progenitors of Type IIn SNe on the other hand lose orders of magnitude more material, $10^{-2}$ to $10^{-1} M_{\odot} \mathrm{yr}^{-1}$ (Kiewe et al. 2010), which result in narrow $\left(\sim 100 \mathrm{~km} \mathrm{~s}^{-1}\right)$ emission lines in their spectra due to ionization of the preexisting CSM which has been excited by the initial flash of the supernova. For example, SN 1998S showed dust formation signatures between days 140 and 268 (Leonard et al. 2000), and SN 2005ip appears to have formed dust both in the cool dense shell (CDS) between days 75 and 150 and then again in the ejecta after day 750 (Smith et al. 2009; Fox et al. 2009). Although not classified as Type IIn, the Type Ib/c SN 2006jc also formed dust in the CDS created by the interaction of the ejecta and the CSM between 50 and 75 days post-explosion (Mattila et al. 2008; Smith et al. 2008) and the Type IIP SN 2007od formed dust sometime between days 120 and 230 through the same mechanism (Andrews et al. 2010).

In this paper, we follow the evolution of the Type IIP SN 2007 it in the optical and IR from day 10 to day 944 . This makes SN 2007it one of only a few Type IIP SNe with longterm and extensive spectral and photometric observations, along with SN 1990E, SN 1999em, SN 1999gi, SN 2002hh, SN 2003gd, SN 2004et, and SN 2005cs (Maguire et al. 2010, and references therein). In Section 3, we discuss the visible lightcurve evolution, including a possible scattered optical light echo seen at late times. In Section 4 we present the optical spectral evolution and describe the unusual spectral evolution of SN 2007it. The IR light-curve evolution is discussed in Section 5, followed by the radiative transfer modeling and spectral energy distribution (SED) fitting in Section 6. In all four sections, we will discuss the evidence that dust has formed in the ejecta of SN 2007it.

\section{OBSERVATIONS AND ANALYSIS}

SN 2007it was discovered in NGC 5530 by R. Evans visually on 2007 September 13 with $V \sim 13.5$ mag. (Evans et al. 2007; Itagaki et al. 2007). Pre-discovery images taken with the All Sky Automated Survey (ASAS-3) constrain the explosion date between 2007 September 4 and 6 (Pojmanski 2007), and for the purposes of this paper we are assuming an explosion date of 2007 September 5 (JD 2454348). It was confirmed spectroscopically to be a Type II SN by the Carnegie Supernova Project on 2007 September 15 (Contreras et al. 2007). Using Tully et al. (2008), which uses distances set by the 2001 Hubble Space Telescope (HST) Cepheid Key Project observations, we are adopting a distance of $11.7 \mathrm{Mpc}$ throughout this paper. However, it should be noted that a previous study done by Tully (1988) had suggested a distance of 16.9 Mpc. We must also note here that no pre-explosion images exist of the host galaxy of SN 2007 it

We have obtained visible spectroscopy and photometry as well as mid-IR photometry of SN 2007it spanning days 107-944. Lists of these observations are presented in Tables 1 and 2. Optical imaging was obtained in Johnson-Cousins BVI 
Table 2

Spectroscopy Summary

\begin{tabular}{lccccc}
\hline \hline Day & JD & Telescope & Instrument & Exposures & Exposure Time (s) \\
\hline 154 & 2454502 & Gemini South & GMOS Spectra & 3 & 900 \\
178 & 2454526 & Gemini South & GMOS Spectra & 3 & 900 \\
209 & 2454557 & Gemini South & GMOS Spectra & 3 & 900 \\
242 & 2454590 & Gemini South & GMOS Spectra & 3 & 900 \\
279 & 2454627 & Gemini South & GMOS Spectra & 3 & 900 \\
302 & 2454650 & Gemini South & GMOS Spectra & 3 & 900 \\
339 & 2454687 & Gemini South & GMOS Spectra & 3 & 900 \\
540 & 2454888 & Gemini South & GMOS Spectra & 3 & 1800 \\
566 & 2454914 & Gemini South & GMOS Spectra & 3 & 1800 \\
589 & 2454937 & Gemini South & GMOS Spectra & 3 & 1800 \\
622 & 2454970 & Gemini South & GMOS Spectra & 3 & 1800 \\
916 & 2455264 & Gemini South & GMOS Spectra & 3 & 1800 \\
\hline
\end{tabular}

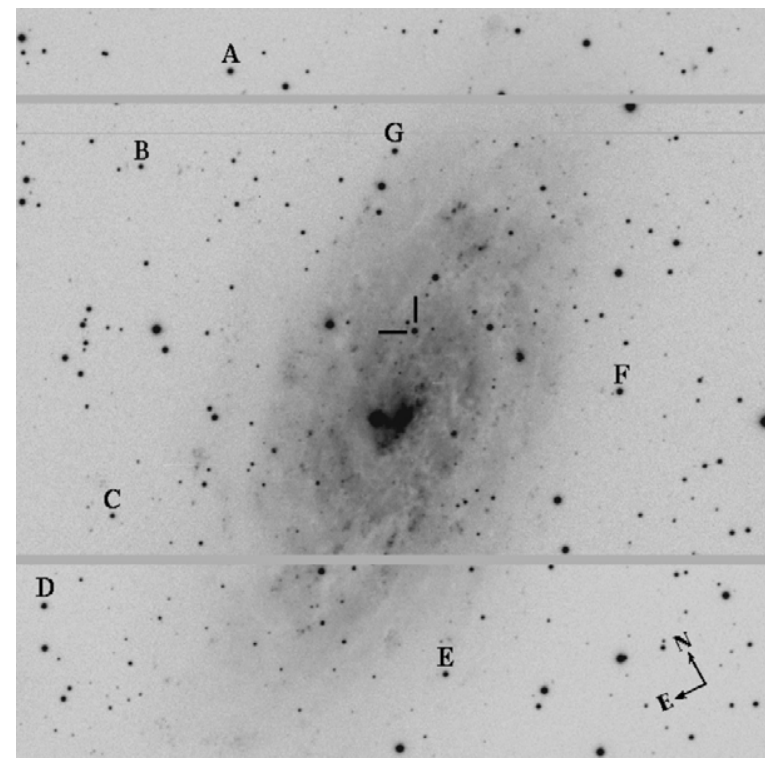

Figure 1. Image of NGC 5530 taken in the $g^{\prime}$ band with Gemini South on 2008 April 12. The tertiary standards listed in Table 3 are labeled alphabetically, and the $\mathrm{SN}$ is indicated at the center of the image. The image is $4.2 \times 4.2$.

with the SMARTS consortium $1.3 \mathrm{~m}$ telescope at Cerro Tololo Inter-American Observatory, Chile. All images were pipeline reduced, shifted, and stacked. Imaging and spectra were also obtained with GMOS/Gemini South (GS-2008A-Q-24, GS2008B-Q-45, GS-2009A-Q-49, GS-2010A-DD-3). The $g^{\prime} r^{\prime} i^{\prime}$ images were reduced and stacked using the IRAF gemini package. The instrumental $g^{\prime} r^{\prime} i^{\prime}$ magnitudes were transformed to standard Johnson-Cousins VRI (Welch et al. 2007; Fukugita et al. 1996). For each night the transformation involved a leastsquares fit with a floating zero point.

One epoch of photometry was obtained with the WFPC2/ PC1 camera on HST in the F450W, F606W, and F814W filters. These images were delivered pipeline reduced, but undrizzled, and stacking and cosmic ray removal was accomplished using the Pyraf task multidrizzle. Transformation into the standard Johnson-Cousins $B V I$ was done using methods outlined by Holtzman et al. (1995). Late-time images were obtained with the Wide Field Camera (WFC) on HST/Advanced Camera for Surveys (ACS) using the F435W, F606W, and F814W filters. These images were pipeline reduced, including drizzling and cosmic ray removal, and transformations to the Johnson-Cousins $B V I$ system were accomplished using methods outlined by Sirianni et al. (2005).

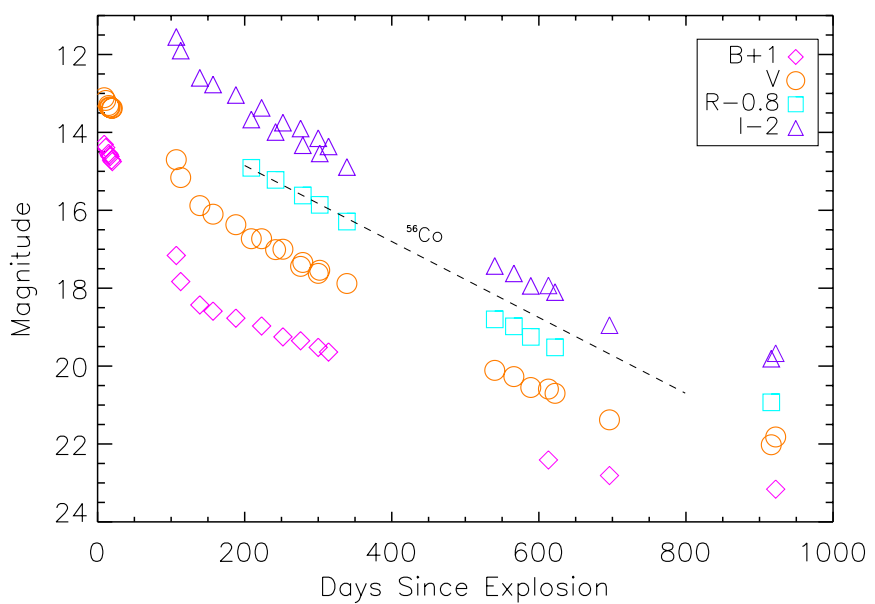

Figure 2. Optical BVRI light curves of SN 2007it. Photometry before day 100 was obtained from Carnegie Supernova Project. The disagreement in values in $I$ between days 200 and 400 is likely due to bandpass differences between SMARTS and Gemini filters.

(A color version of this figure is available in the online journal.)

A BVRI photometric sequence of tertiary standard stars (shown in Figure 1) was derived for the SN 2007it field, using the same method as Andrews et al. (2010). The BVRI magnitudes for these standards are located in Table 3 . The $B V R I$ light curves of SN 2007it are shown in Figure 2 and listed in Table 4 and the absolute $V$ magnitude is shown in comparison with other similar Type II SNe in Figure 3. Early-time photometry of SN 2007it from days 9-20 (shown in Figures 2 and 3) was obtained from the Carnegie Supernova Project. The discrepancies between the SMARTS and Gemini I magnitudes are likely due to the bandpass of the Cousins I filter covering part of the Ca II IR-triplet around $8500 \AA$ that is not covered by the Gemini $i^{\prime}$ filter. This would cause the SMARTS photometry to appear brighter due to the added Ca II flux. Uncertainties for the Gemini photometry were calculated by adding in quadrature the transformation uncertainty quoted in Welch et al. (2007), photon statistics, and the zero point deviation of the standard stars for each epoch. The HST uncertainties consist of the transformation uncertainty from Holtzman et al. (1995) or Sirianni et al. (2005) and photon statistics added in quadrature.

For each GMOS/Gemini South epoch, three spectra of 900s (before day 540) or $1800 \mathrm{~s}$ (day 540 and after) were obtained in long-slit mode using grating B600 and a slit width of 0 ':75. Central wavelengths of 5950, 5970, and $5990 \AA$ were chosen to prevent important spectral features from falling on 
Table 3

Tertiary BVRI Standards for NGC 5530

\begin{tabular}{lcccc}
\hline \hline Star & $B$ & $V$ & $R$ & $I$ \\
\hline A & $17.743 \pm 0.012$ & $17.060 \pm 0.001$ & $16.655 \pm 0.012$ & $16.272 \pm 0.017$ \\
B & $19.115 \pm 0.039$ & $17.667 \pm 0.011$ & $16.696 \pm 0.015$ & $15.648 \pm 0.013$ \\
C & $19.235 \pm 0.040$ & $18.099 \pm 0.015$ & $17.485 \pm 0.014$ & $16.945 \pm 0.025$ \\
D & $18.057 \pm 0.014$ & $17.254 \pm 0.021$ & $16.772 \pm 0.033$ & $16.209 \pm 0.088$ \\
E & $18.652 \pm 0.029$ & $17.219 \pm 0.016$ & $16.240 \pm 0.007$ & $15.154 \pm 0.011$ \\
F & $17.672 \pm 0.012$ & $16.818 \pm 0.009$ & $16.318 \pm 0.008$ & $15.812 \pm 0.014$ \\
G & $18.281 \pm 0.017$ & $17.587 \pm 0.033$ & $17.205 \pm 0.020$ & $16.778 \pm 0.028$
\end{tabular}

Table 4

Optical Photometry of SN 2007it

\begin{tabular}{|c|c|c|c|c|}
\hline Day & $B$ & $V$ & $R$ & $I$ \\
\hline 107 & $16.16 \pm 0.10$ & $14.70 \pm 0.04$ & & $13.55 \pm 0.04$ \\
\hline 113 & $16.83 \pm 0.11$ & $15.16 \pm 0.08$ & $\ldots$ & $13.90 \pm 0.06$ \\
\hline 139 & $17.43 \pm 0.04$ & $15.88 \pm 0.03$ & $\ldots$ & $14.60 \pm 0.03$ \\
\hline 157 & $17.59 \pm 0.04$ & $16.1 \pm 0.03$ & $\ldots$ & $14.77 \pm 0.03$ \\
\hline 188 & $17.77 \pm 0.05$ & $16.37 \pm 0.02$ & $\ldots$ & $15.04 \pm 0.03$ \\
\hline 209 & $\ldots$ & $16.73 \pm 0.04$ & $15.71 \pm 0.03$ & $15.67 \pm 0.03$ \\
\hline 223 & $17.97 \pm 0.03$ & $16.73 \pm 0.03$ & $\ldots$ & $15.37 \pm 0.03$ \\
\hline 242 & $\ldots$ & $17.01 \pm 0.04$ & $16.02 \pm 0.02$ & $15.99 \pm 0.03$ \\
\hline 252 & $18.52 \pm 0.05$ & $17.00 \pm 0.03$ & $\ldots$ & $15.75 \pm 0.09$ \\
\hline 276 & $18.35 \pm 0.04$ & $17.23 \pm 0.03$ & $\ldots$ & $15.90 \pm 0.03$ \\
\hline 279 & $\ldots$ & $17.34 \pm 0.04$ & $16.42 \pm 0.02$ & $16.33 \pm 0.03$ \\
\hline 300 & $18.52 \pm 0.05$ & $17.44 \pm 0.03$ & $\ldots$ & $16.15 \pm 0.03$ \\
\hline 302 & & $17.54 \pm 0.04$ & $16.66 \pm 0.03$ & $16.54 \pm 0.02$ \\
\hline 314 & $18.64 \pm 0.05$ & $17.62 \pm 0.05$ & $\ldots$ & $16.36 \pm 0.04$ \\
\hline 339 & $\ldots$ & $17.88 \pm 0.04$ & $17.09 \pm 0.04$ & $16.89 \pm 0.05$ \\
\hline 540 & $\ldots$ & $20.11 \pm 0.04$ & $19.60 \pm 0.03$ & $19.43 \pm 0.03$ \\
\hline 566 & $\ldots$ & $20.27 \pm 0.04$ & $19.78 \pm 0.04$ & $19.62 \pm 0.05$ \\
\hline 589 & $\ldots$ & $20.55 \pm 0.04$ & $20.05 \pm 0.02$ & $19.94 \pm 0.03$ \\
\hline 613 & $21.41 \pm 0.16$ & $20.59 \pm 0.11$ & $\ldots$ & $19.93 \pm 0.09$ \\
\hline 622 & & $20.7 \pm 0.04$ & $20.32 \pm 0.03$ & $20.10 \pm 0.03$ \\
\hline 696 & $21.81 \pm 0.12$ & $21.38 \pm 0.05$ & $\ldots$ & $20.95 \pm 0.05$ \\
\hline 916 & & $22.02 \pm 0.03$ & $21.73 \pm 0.03$ & $21.81 \pm 0.04$ \\
\hline 922 & $22.16 \pm 0.18$ & $21.82 \pm 0.10$ & $\ldots$ & $20.95 \pm 0.14$ \\
\hline
\end{tabular}

chip gaps. A $2 \times 2$ binning in the low gain setting was used. Spectra were reduced using the IRAF gemini package. The sky subtraction regions were determined by visual inspection to prevent contamination from material not associated with the $\mathrm{SN}$, and the spectra were extracted using 15 rows centered on the SN. The spectra from each individual night were averaged and have been corrected for the radial velocity of NGC 5530 $\left(1196 \mathrm{~km} \mathrm{~s}^{-1}\right)$. They are presented in Figures 4 and 5 .

In order to estimate the reddening toward SN 2007it, we compared the day 10 spectrum with other Type IIPs of a similar epoch with known $E(B-V)$ values. The day 10 spectrum was most similar to SN 2007od and SN 1999em. Given the quoted foreground reddening of $A_{v}=0.39$ from NED for SN 2007it, and the total reddening by $0.39 \pm 0.04$ of SN 2007od (Andrews et al. 2010) and 0.31 \pm 0.16 for SN 1999em (Maguire et al. 2010, and references therein), we have estimated that the reddening of the host galaxy is relatively low, and adopt $A_{v}=0.39$ for the purpose of this paper. We also first attempted to use the narrow $\mathrm{Na}$ I D absorption seen in the day 10 spectrum of SN 2007 it to determine the foreground extinction. The equivalent width (EW) of the blended Na I D lines was $1.6 \pm 0.1 \mathrm{~A}$, which corresponded to an $A_{v} \sim 1.27 \pm 0.1$ of the host galaxy (Barbon et al. 1990). This value does not agree with the day 10 spectrum which indicated that the $\mathrm{SN}$ is not reddened by a high amount. Discrepancies between reddening measured from Na I EW and actual values are not unique. For example, Blondin et al. (2009)

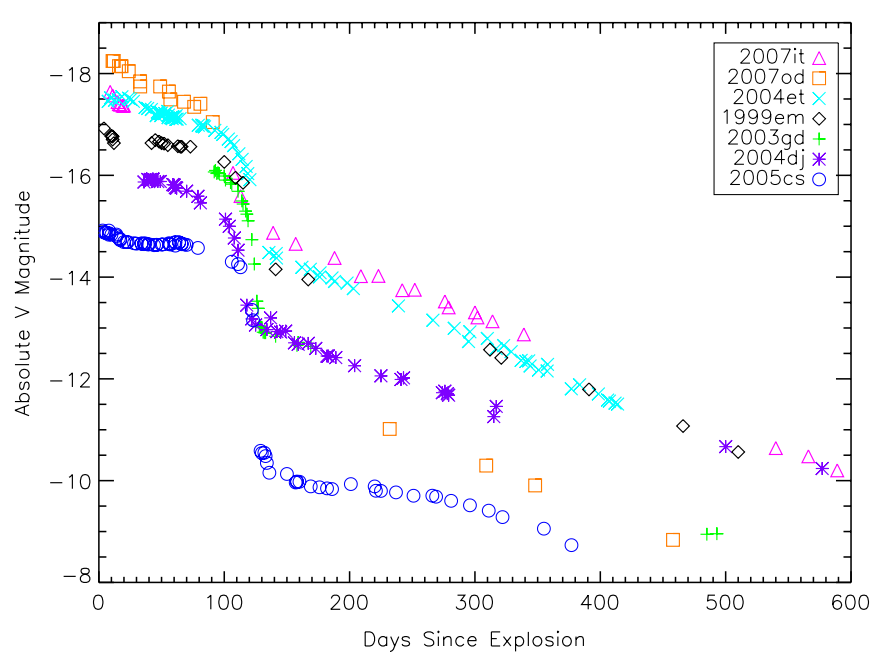

Figure 3. Absolute $V$ light curves of a sample of Type IIP SNe. All SNe have been corrected for reddening and the data are from SN 2007od (Andrews et al. 2010), SN 2004et (Kotak et al. 2009; Misra et al. 2007), SN 1999em (Elmhamdi et al. 2003), SN 2003gd (Hendry et al. 2005), SN 2004dj (Vinkó et al. 2006; Zhang et al. 2006), and SN 2005cs (Pastorello et al. 2009).

(A color version of this figure is available in the online journal.)

found that for an Na I EW of $\sim 0.2 \AA \quad E(B-V)$ had values ranging between 0.2 and 1.5 , and that one could not solely rely on EW values. Therefore, we do not use the NaI reddening estimate in this paper.

The Spitzer IRAC (3.6, 4.5, 5.8, and $8.0 \mu \mathrm{m})$ images were mosaicked and resampled using standard MOPEX procedures to improve photometric quality. Point spread function photometry was performed using the position specific point response function images. Table 5 contains the measured mid-IR fluxes obtained from Spitzer. Figure 6 presents the SED of SN 2007it at four epochs: Spitzer (day 351)/Gemini (day 339), Spitzer (day 561)/Gemini (day 566), Spitzer (day 718)/HST (day 696), and Spitzer (day 944)/Gemini (day 916). Statistical uncertainties presented in the plot represent $1 \sigma$ errors. The data have been corrected for foreground extinction.

\section{LIGHT-CURVE EVOLUTION}

\subsection{Day 9-Day 339}

The photometric evolution of SN 2007it at early times seems to be consistent with other Type IIP supernova light curves, as can be seen in Figure 3. The plateau phase appears to begin near day 20 , and lasts until around the time that the SN becomes visible again on day 107 when it is in the midst of its post-plateau drop in brightness. Therefore, the plateau phase lasted $\sim 80$ days. Given the distance to SN 2007it and the $V$ magnitudes on day 20 and day 107, the average absolute magnitude of SN 2007it on day $\sim 50$ is estimated to be $M_{V}=$ $-16.7\left(L=1.6 \times 10^{42} \mathrm{erg} \mathrm{s}^{-1}\right)$, once corrections for reddening have been made. Comparing these values with other Type IIP SNe (Maguire et al. 2010, and references therein) indicates that SN 2007it is one of the more luminous, well-studied Type IIP $\mathrm{SNe}$ to date and is quite similar to SN 2004et (see Figure 3).

After the plateau phase, the drop to the radioactive tail is roughly 2 mag. Type IIP SN normally experience a decrease in luminosity of $\sim 1.5-3$ mag based upon the amount of ${ }^{56} \mathrm{Ni}$ present (Woosley \& Weaver 1986; Kasen \& Woosley 2009). For comparison, SN 2007it shows roughly the same plateau duration and drop as SN 2004et (Figure 3), which may suggest a similar 


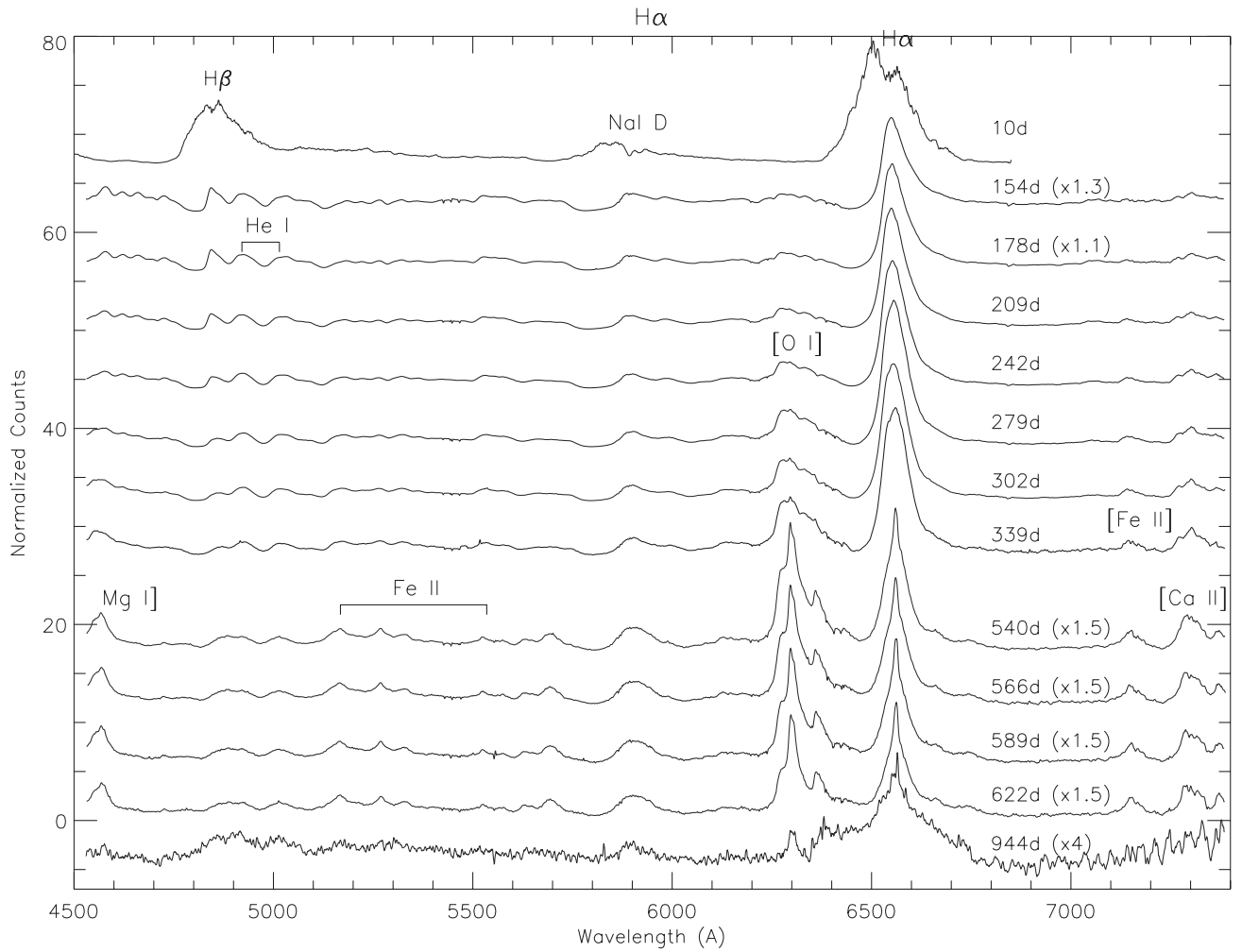

Figure 4. Spectral evolution of SN 2007it from day 10 to day 916. All spectra other than day 10 are from Gemini/GMOS. Of interest is the brightness of the [O I] $\lambda \lambda$ 6300,6363 lines in relation to $\mathrm{H} \alpha$ and [Ca II] lines.

Table 5

Spitzer Photometry of SN 2007it

\begin{tabular}{lcccc}
\hline \hline Day & $3.6 \mu \mathrm{m}(\mu \mathrm{Jy})$ & $4.5 \mu \mathrm{m}(\mu \mathrm{Jy})$ & $5.8 \mu \mathrm{m}(\mu \mathrm{Jy})$ & $8.0 \mu \mathrm{m}(\mu \mathrm{Jy})$ \\
\hline 351 & $257.28 \pm 6.64$ & $625.75 \pm 12.10$ & $317.40 \pm 13.16$ & $363.41 \pm 23.28$ \\
561 & $528.69 \pm 11.69$ & $600.90 \pm 11.07$ & $660.69 \pm 17.65$ & $628.06 \pm 23.14$ \\
718 & $213.21 \pm 6.11$ & $354.16 \pm 7.15$ & $\ldots$ & $\ldots$ \\
944 & $39.12 \pm 2.30$ & $142.96 \pm 3.28$ & $\cdots$ & $\cdots$ \\
\hline
\end{tabular}

${ }^{56} \mathrm{Ni}$ mass. This is discussed in more detail below. The decline in luminosity then follows the ${ }^{56} \mathrm{Co}$ decay closely (see Figure 2), until the $\mathrm{SN}$ is lost behind the sun after day 339.

\subsection{Day 509-Day 922}

Once SN 2007it was observed again on day 509, we found that its brightness had fallen below the expected ${ }^{56} \mathrm{Co}$ decay by $\sim 0.5$ mag in $R$. The $R$ band contains the $\mathrm{H} \alpha$ and [O I] $\lambda \lambda 6300,6364$ emission features, which are responsible for most of the flux at late times. The most likely cause for this decreased decline in brightness is new dust formation between days 339 and 509. This is the time period during which dust typically condenses in the ejecta of SNe. After this drop, the ${ }^{56}$ Co decay was maintained, indicating that little or no further dust formation occurred after day 509. Other dust indicators such as the simultaneous increase in the IR luminosity and red-wing attenuation of the [O I] lines, discussed below, indicate that this is indeed the case.

Also of note is another deviation from the ${ }^{56} \mathrm{Co}$ decay curve seen as a flattening of the light curve after day 700. This was also seen in SN 2007od (Andrews et al. 2010), SN 2003gd (Sugerman 2005), and SN 2002hh (Welch et al. 2007), and is likely a light echo caused by dust reflecting light from the flash of the SN explosion, keeping the luminosity brighter than expected at late times. A comprehensive explanation of scattered light echoes is presented in Sugerman (2003). Scattered light echoes are expected to become major contributors to the emission of $\mathrm{SNe}$ around 8-9 mag below maximum (Patat 2005), which is when we see the flattening of the SN 2007it light curve. Analysis of this echo is beyond the scope of this paper, and will be presented in a forthcoming paper. Subtraction of images of SN 2007it taken with HST/ACS on day 922 and three epochs of WFC3 data (to be presented in another paper) between days 857 and 1009 revealed a residual in the southwest corner roughly 4 pixels from the center of the SN corresponding to an echo from ISM material 21 pc away from the SN (J. E. Andrews et al. 2011, in preparation). Comparison of photometry from early times to the late-time flattening show that the late-time color is much bluer, with $B-V$ dropping from $\sim 1.5$ on day 107 to $\sim 0.3$ on day 922 . This will happen when the CSM or ISM dust is preferentially scattering the blue light as was seen in SN 2006gy on day 810 (Miller et al. 2010). The resolved light echo, along with the blue late-time colors, indicate that the flattening of the light curve after day 696 is likely due to a light echo and not CSM interaction at late times.

\section{SPECTRAL EVOLUTION}

Figure 4 shows the optical spectral evolution of SN 2007it from day 10 to day 922 . The early time spectra show broad 


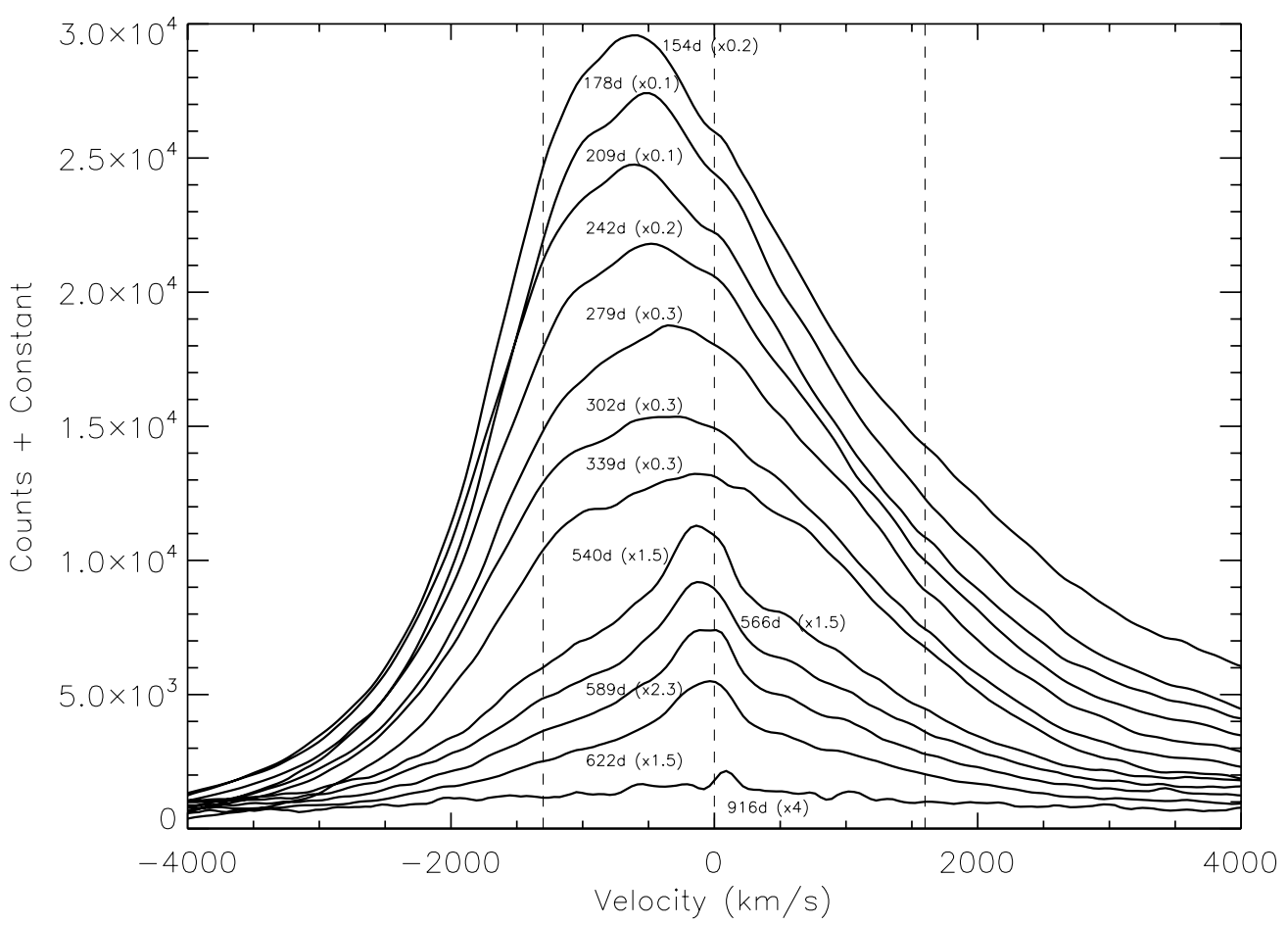

Figure 5. H $\alpha$ evolution from day 154 (top) to day 916 (bottom). Notice the emergence of an intermediate width component starting on day 540 which may indicate CSM interaction.

$\mathrm{H} \alpha$ and $\mathrm{H} \beta$ emission and strong $\mathrm{Na}$ I D absorption. The $\mathrm{H} \alpha$ evolution is seen in Figure 5 and shows a blueshift for at least the first 200 days. Blueshifted $\mathrm{H} \alpha$ emission has been seen in other Type II SNe such as SN 1988A (Turatto et al. 1993), SN 1998A (Pastorello et al. 2005), and SN 1999em (Elmhamdi et al. 2003). Chugai (1988b) and later Jeffery \& Branch (1990) proposed that this was caused by the reflection of photons by the photosphere. For 1988A and 1999em, the blueshift only lasted $\sim 80$ days, whereas SN 2007it and SN 1998A seem to have a blueshift persisting well past 150 days. By day 200, when the SN is well into the nebular phase, strong [O I] $\lambda \lambda 6300,6363$ emission is seen (shown in Figure 5), as well as [Ca II] $\lambda \lambda 7291,7324$ and [Fe II $] \lambda 7155$ emission.

Once we recover SN 2007it at day 509, the [O I] line strengths rival that of $\mathrm{H} \alpha$, while the [CaII] lines are at most, half the strength of $\mathrm{H} \alpha$. This is unusual for Type IIP SNe, since most exhibit stronger [Ca II] than [O I]. The only other example of a Type II SN with similar late-time line strengths as SN 2007it was the Type IIL SN 1970G (Pronik et al. 1976; Chugai 1988a). Unfortunately no optical spectra were taken after $\sim$ day 350 of SN 1970G to allow further comparisons of the spectral evolution. There is also an emission feature emerging after day 200 at $7380 \AA$ which is separate from the [Ca II $] /[\mathrm{O}$ II $]$ blended lines, and could be a [Ni II] $\lambda 7380$ line which was also identified in the Type IIn SN 2006gy (Kawabata et al. 2009), Type Ic SN 2006aj (Maeda et al. 2007), and a few Type Ia SNe (Maeda et al. 2010). This line may be present in other SNe but hidden by strong [Ca II] emission (Maeda et al. 2007).

Of special note are the multi-component peaks of the [OI] $\lambda \lambda 6300,6364$ lines that are visible in our day 154 spectra and persist to day 622 (Figure 6). These emission line profiles are similar to the multiple peaks seen in the $\mathrm{H} \alpha$ emission of $\mathrm{SNe}$ 1998S (Leonard et al. 2000) and 2007od (Andrews et al. 2010). Both the 6300 and $6364 \AA$ lines have a blueshifted component occurring at $-1300 \mathrm{~km} \mathrm{~s}^{-1}$ in relation to the component seen at the rest velocity of the galaxy. There seems to be no corresponding red peaks at $+1300 \mathrm{~km} \mathrm{~s}^{-1}$. Because CSM interaction is normally only seen in $\mathrm{H}$ and $\mathrm{He}$ lines, this suggests another origin for the multiple peaks. Similar asymmetries in the [OI] lines have been seen in numerous H-poor CCSNe (Type Ib, Ic, and IIb), for example, SNe 2005aj, 2006T, and 2008ax, but seems to be rare in normal Type IIP (Modjaz et al. 2008; Taubenberger et al. 2009; Milisavljevic et al. 2010). These asymmetries can be seen approximately 2 months postexplosion in H-poor CCSNe, but due to the lack of spectral observations between days 10 and 154 in SN 2007it, we cannot pinpoint the exact date before day 154 when the [O I] lines and the corresponding asymmetries appeared. Milisavljevic et al. (2010) suggest that the absence of a red component combined with the presence of blue peak the same distance away from both the 6300 and $6364 \AA$ lines could be explained by emission from an asymmetric blob of $\mathrm{O}$ material ejected on the forward side of the SN during the explosion. They do caution that a torus of $\mathrm{O}$ material is less likely but cannot be ruled out, and that the redshifted components could exist but are hidden either by the SN orientation or obscuring dust. The existence of fast moving knots seen in oxygen-rich SN remnants such as Puppis $\mathrm{A}$ and Cas A may substantiate the asymmetric blob scenario. According to Winkler \& Kirshner (1985), it is likely that these knots are intact fragments of the core of the progenitor star. These remnant oxygen knots have radial velocities consistent with the blue [O I] components of SN 2007it.

Signatures of CSM interaction can also be seen in the $\mathrm{H} \alpha$ and [O I] lines (Figures 5 and 6). The CSM interaction is manifested as a narrower feature (FWHM $\sim 1000 \mathrm{~km} \mathrm{~s}^{-1}$ ) at the rest velocity rising above the broader (FWHM $\sim 4000 \mathrm{~km} \mathrm{~s}^{-1}$ ) ejecta base. Although this feature does not become apparent until day 509 in the $\mathrm{H} \alpha$ spectra, it seems to be present since day 154 in the [O I] emission. We then surmise that the intermediate peak was present in both lines since day 154 , but the overwhelming ejecta 

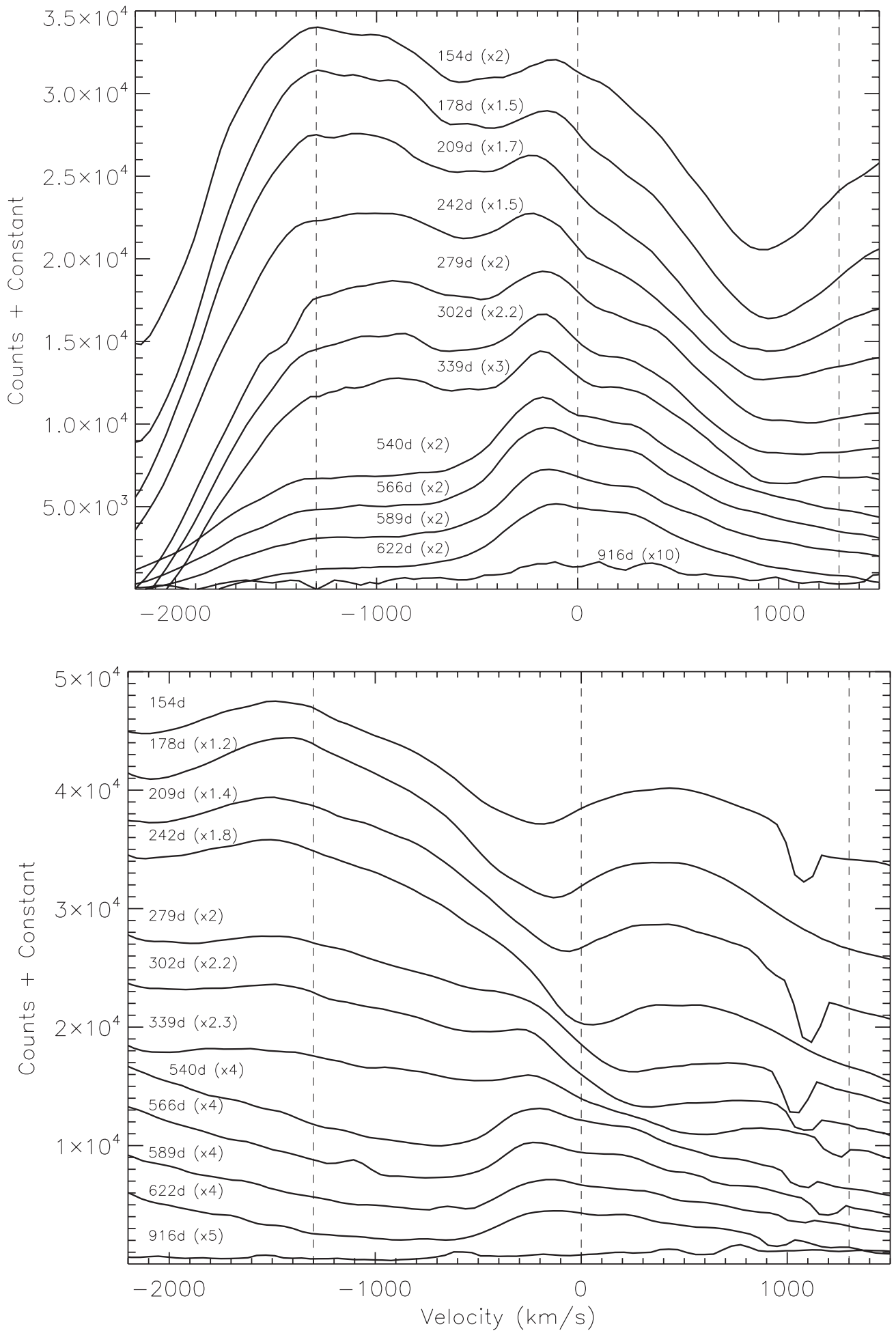

Figure 6. Evolution of [O I] $6300 \AA$ (top) and $6364 \AA$ (bottom) lines from day 154 to day 916 . There is a separate blueshifted component at $-1300 \mathrm{~km} \mathrm{~s}{ }^{-1}$ from each [O I] line, which we believe is created by a torus of oxygen material surrounding the SN or a blob of O-rich ejecta on the forward side of the SN.

emission in $\mathrm{H} \alpha$ kept it hidden until day 509. From the day 154 spectra and onward in [O I] there also seems to be an attenuation of the red-wing of the central peak, especially in $6300 \AA$ which becomes even more apparent after day 509 when the asymmetric blob emission has faded. There are two possibilities for these asymmetries, attenuation from pre-existing dust or Bochum events like that seen in $\mathrm{H} \alpha$ of SNe 1987A and 1999em. For a full discussion on these events see Elmhamdi et al. (2003). The shape of the [O I] central peaks are similar to modeled profiles presented in Elmhamdi et al. (2003), where the peak has been shifted toward the blue side due to the presence of dust in the ejecta. As we will show below, IR data from day 351 suggests the presence of an IR echo from flash-heated pre-existing dust. This pre-existing dust is likely the cause of the attenuation seen in the [O I] lines. We do not see signatures of new dust forming from asymmetries in the spectral lines, although we must point 
out that it is possible that if the new dust is concentrated in a ring or torus around the SN, newly formed dust could be hidden by the viewing angle. If the newly formed dust has formed in the CDS between the forward and reverse shocks formed in the CSM interaction, the ejecta emission would be located interior to the newly formed dust, which would allow equal attenuation of the blue and red emission.

\subsection{Mass Estimates}

We estimate a ${ }^{56} \mathrm{Ni}$ mass for SN 2007it of $M_{\mathrm{Ni}}=0.09_{-0.02}^{+0.01} M_{\odot}$ from the methods of Hamuy (2003). The bolometric luminosity of the radioactive tail (in erg s $\mathrm{s}^{-1}$ ) is calculated as

$$
\log _{10} L_{t}=\frac{-\left[V_{t}-A(V)+\mathrm{BC}\right]+5 \log _{10} D-8.14}{2.5}
$$

with $A_{V}=0.39 \mathrm{mag}$, and a bolometric correction of $\mathrm{BC}=0.26$. The nickel mass is then calculated at various times during this phase as

$$
M_{\mathrm{Ni}}=\left(7.866 \times 10^{-44}\right) L_{t} \exp \left[\frac{\frac{t_{t}-t_{0}}{1+z}-6.1}{111.26}\right] M_{\odot} .
$$

Here, 6.1 and 111.26 days are the half-lives of ${ }^{56} \mathrm{Ni}$ and ${ }^{56} \mathrm{Co}$, respectively, and $t_{t}-t_{0}$ is the age of the $\mathrm{SN}$. This calculated $\mathrm{Ni}$ mass is similar, if slightly higher, than other Type IIP SNe. SN 2004et has an estimated $M_{\mathrm{Ni}}$ mass of $0.056 M_{\odot}$ (Maguire et al. 2010), SNe 1999em, 2003gd, and 2004dj each have $\sim 0.02 M_{\odot}$ (Elmhamdi et al. 2003; Hendry et al. 2005; Vinkó et al. 2006), and the low-luminosity SN 2005 cs contains $3 \times 10^{-3} M_{\odot}$ of ${ }^{56} \mathrm{Ni}$ (Pastorello et al. 2009).

We have estimated the mass of oxygen by two separate ways using the strength of the [OI] doublet in the nebular phase before the onset of dust formation. Using a temperature range of $3500-4000 \mathrm{~K}$ and a flux $\left(F_{\left[\mathrm{O}_{\mathrm{I}}\right]}\right)$ of $9.7 \times 10^{-14} \mathrm{erg} \mathrm{s}^{-1} \mathrm{~cm}^{-2}$ for the day 286 spectrum (obtained from the Carnegie Supernova Project), we estimate $M_{\mathrm{O}}=0.2-0.9 M_{\odot}$ using

$$
M_{[\mathrm{OI}]}=10^{8} F_{[\mathrm{OI}]} D^{2} e^{2.28 / T_{4}},
$$

which is Equation (1) in Uomoto (1986). As a second method we implemented the relationship given in Elmhamdi et al. (2003),

$$
L_{\left[O_{\mathrm{I}}\right]}=\eta \frac{M_{\mathrm{O}}}{M_{\mathrm{exc}}} L_{\mathrm{CO}},
$$

under the assumption that SN 2007it had the same $\eta$ (the efficiency of energy transfer from $\mathrm{O}$ mass to [O I] doublet luminosity) and excited mass as SN 1987A, and that the $L_{\mathrm{Co}}$, the luminosity of the Co emission, was directly related to the mass of ${ }^{56} \mathrm{Ni}$. Once again we used an oxygen flux of $9.7 \times$ $10^{-14} \mathrm{erg} \mathrm{s}^{-1} \mathrm{~cm}^{-2}$. This calculation gives us an oxygen mass of 0.96 that of SN 1987A, or between 1.15 and $1.44 M_{\odot}$. These values, although not consistent with each other, are very similar to the values calculated for SN 2004et in Maguire et al. (2010).

Comparing the relationship between ${ }^{56} \mathrm{Ni}$ and progenitor mass in other SNe (for example, Table 5 in Misra et al. 2007), it is likely that SN 2007it had a main sequence mass $\geqslant 20 M_{\odot}$. Recently, Dessart et al. (2010) have suggested that using the velocity of the $\mathrm{H} \alpha$ line at $\sim$ day 15 and the velocity of the $\left[\mathrm{O}_{\mathrm{I}}\right] \lambda \lambda 6300,6363$ lines at $\sim 300$ days could be an indicator of progenitor ZAMS mass. Using the values of $10,960 \mathrm{~km} \mathrm{~s}^{-1}$ and $2000 \mathrm{~km} \mathrm{~s}^{-1}$, respectively, and the estimated mass of oxygen from above suggests a progenitor mass between 16 and $20 M_{\odot}$.

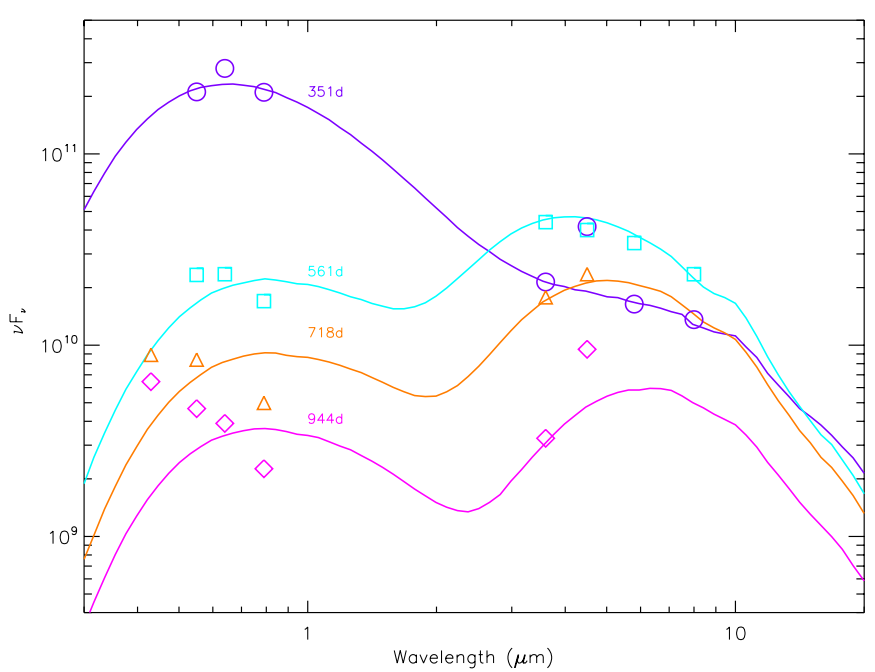

Figure 7. MOCASSIN fits from our smooth shell modeling scenario (Table 6) for days 351 (circles), 561 (squares), 718 (triangles), and 944 (diamonds). The optical points are from day 339, 566, 696, and 916/922 (Table 4), and have been corrected for foreground extinction. Error bars are equal to or smaller than symbol size. On days 718 and 944, a large portion of the optical luminosity is likely derived from the light echo, which due to its scattering nature will make the SN appear to be more blue (Patat 2005). The clumpy shell and torus modeling is consistent with the smooth shell fits, and are not shown.

(A color version of this figure is available in the online journal.)

Fransson \& Chevalier $(1987,1989)$ have also suggested that the ratio between $[\mathrm{Ca} \mathrm{II}] /\left[\mathrm{O}_{\mathrm{I}}\right]$ lines in the nebular phase can be an indicator of progenitor mass, with smaller ratios belonging to higher mass progenitors. This ratio is 0.7 for SN 2007it between days 209 and 351, which is quite small for a Type IIP SN. Elmhamdi et al. (2004) showed that SN with ratios similar to SN 2007it is normally of Type Ib/c. SN 1987A had a ratio of $\sim 3$ from day 250-450 and SN 2005cs meanwhile showed a ratio of $4.2 \pm 0.6$ on day 334 (Pastorello et al. 2009), which is $40 \%$ larger than SN 1987A, and over six times greater than SN 2007it. This may indicate that the progenitor of SN 2007it may have been more massive than either SN 2005cs (7-13 $\left.M_{\odot}\right)$ or SN 1987A (14-20 $\left.M_{\odot}\right)$, with roughly a mass of 20-27 $M_{\odot}$. Even though each method yields different dust masses, and uncertainties in distance and reddening may add more ambiguity to these estimates, they all suggest that SN 2007it had a progenitor mass of $\sim 20_{-4}^{+7} M_{\odot}$. Unfortunately, no images of the progenitor star exist, although as was shown in the case for SN 2004et, masses from progenitor images do not necessarily always agree with other estimates from explosion parameters (Crockett et al. 2011; Maguire et al. 2010, and references therein).

\section{MID-IR EVOLUTION}

SN 2007it was monitored in the mid-IR for four epochs spanning days 351-944 (see Table 5). As can be seen in Figure 7, the first epoch on day 351 shows an indication of an increased flux in the $4.5 \mu \mathrm{m}$ band with respect to the other channels. This elevated emission in this band photometry was also seen around the same time period in SNe 2005af (Kotak et al. 2006), 2004dj (Kotak et al. 2005; Szalai et al. 2011), and 2004et (Kotak et al. 2009), and has been attributed to $\mathrm{CO}$ fundamental band emission. Less prominent is the possible extra emission at $8.0 \mu \mathrm{m}$ which may be due to $\mathrm{SiO}$ emission. SiO emission was discovered in 1987A (Roche et al. 1991) and also in SN 2004et (Kotak et al. 2009) and 2005af (Kotak et al. 2006). $\mathrm{CO}$ and $\mathrm{SiO}$ are thought to be 
Table 6

Monte Carlo Radiative Transfer Models

\begin{tabular}{lcccccccc}
\hline \hline Epoch & \multirow{2}{*}{$\mathrm{AC} / \mathrm{Si}$} & \multicolumn{9}{c}{ Smooth } & \multicolumn{2}{c}{ Clumpy } \\
\cline { 3 - 8 } & & $T_{\mathrm{ej}}(\mathrm{K})$ & $R_{\text {in }}(\mathrm{cm})$ & $R_{\text {out }}(\mathrm{cm})$ & $L_{\text {tot }}\left(L_{\odot}\right)$ & $\tau_{v}$ & $M_{d}\left(M_{\odot}\right)$ & $M_{d}\left(M_{\odot}\right)$ \\
\hline $351 \mathrm{~d}$ & $0.75 / 0.25$ & 5700 & $3.2 \mathrm{e} 16$ & $9.0 \mathrm{e} 17$ & $1.9 \mathrm{e} 7$ & 0.08 & $5.0 \mathrm{e}-4$ & $7.3 \mathrm{e}-4$ \\
$561 \mathrm{~d}$ & $0.75 / 0.25$ & 5700 & $7.0 \mathrm{e} 15$ & $2.0 \mathrm{e} 16$ & $4.6 \mathrm{e} 6$ & 1.29 & $7.0 \mathrm{e}-5$ & $1.0 \mathrm{e}-4$ \\
$718 \mathrm{~d}$ & $0.75 / 0.25$ & 5700 & $7.0 \mathrm{e} 15$ & $2.0 \mathrm{e} 16$ & $2.4 \mathrm{e} 6$ & 1.4 & $8.0 \mathrm{e}-5$ & $1.3 \mathrm{e}-4$ \\
$944 \mathrm{~d}$ & $0.75 / 0.25$ & 5700 & $7.0 \mathrm{e} 15$ & $2.0 \mathrm{e} 16$ & $6.9 \mathrm{e} 5$ & 0.98 & $4.6 \mathrm{e}-5$ & $1.3 \mathrm{e}-4$ \\
\hline
\end{tabular}

Table 7

Monte Carlo Radiative Transfer Torus Models

\begin{tabular}{lccccccc}
\hline \hline Epoch & $\mathrm{AC} / \mathrm{Si}$ & $T_{\mathrm{ej}}(\mathrm{K})$ & $R_{\text {in }}(\mathrm{cm})$ & $R_{\text {out }}(\mathrm{cm})$ & $L_{\text {tot }}\left(L_{\odot}\right)$ & $\tau_{v}$ & $M_{d}\left(M_{\odot}\right)$ \\
\hline $351 \mathrm{~d}$ & $0.75 / 0.25$ & 5700 & $3.2 \mathrm{e} 16$ & $2.5 \mathrm{e} 17$ & $1.9 \mathrm{e} 7$ & 0.08 & $1.6 \mathrm{e}-4$ \\
$561 \mathrm{~d}$ & $0.75 / 0.25$ & 5700 & $7.0 \mathrm{e} 15$ & $2.4 \mathrm{e} 16$ & $4.6 \mathrm{e} 6$ & 1.27 & $7.9 \mathrm{e}-5$ \\
$718 \mathrm{~d}$ & $0.75 / 0.25$ & 5700 & $7.0 \mathrm{e} 15$ & $2.4 \mathrm{e} 16$ & $2.4 \mathrm{e} 6$ & 1.27 & $7.9 \mathrm{e}-5$ \\
$944 \mathrm{~d}$ & $0.75 / 0.25$ & 5700 & $7.0 \mathrm{e} 15$ & $2.4 \mathrm{e} 16$ & $6.9 \mathrm{e} 5$ & 1.27 & $7.9 \mathrm{e}-5$ \\
\hline
\end{tabular}

precursors to dust formation (Nozawa et al. 2003) since they likely provide the molecular foundation from which the dust grains form. Therefore, the presence of these molecules is important in understanding the whole story of dust formation in $\mathrm{SNe}$.

Day 351 shows the presence of an IR excess, well before the estimated time of new dust formation as indicated by visible photometry. A likely cause for this elevated flux is an IR echo from flash-heated pre-existing CSM dust. Comparison with the models of SN 1980K presented in Dwek (1983), which also had a peak luminosity of $\sim 10^{9} L_{\odot}$, indicates a dust-free cavity extending to $3 \times 10^{17} \mathrm{~cm}$ with a shell of material extending up to or past $6.3 \times 10^{17} \mathrm{~cm}$. This corresponds to a plateau of luminosity lasting from day $\sim 200$ up to day 643, depending on the model. We do not have any IR observations prior to day 351 , so we cannot definitively suggest the early behavior, but it is likely that an IR excess was present early on and we are seeing the continued plateau of this heated dust on day 351, similar to that seen in SN 1980K. If, though, we use the optical spectra on day 339 before the CSM interaction is apparent in $\mathrm{H} \alpha$ and day 540 when the intermediate peak is visible along with the ejecta velocity on day 10 of $10,960 \mathrm{~km} \mathrm{~s}^{-1}$ (Contreras et al. 2007), we estimate that the initial SN flash has cleared a dust-free cavity between 3.2 and $5.3 \times 10^{16} \mathrm{~cm}$ from the center. This agrees with estimates for SN 2005ip, which has the same $L_{\text {peak }} \sim 10^{9} L_{\odot}$, and a vaporization radius of $\sim 10^{16} \mathrm{~cm}$ (Fox et al. 2010). Assuming a cavity was cleared out to $4 \times 10^{16} \mathrm{~cm}$, the optical and UV light would reach this boundary at around 15 days and the time, $t$, for the IR plateau to last would only be $2 R / c$ or $\sim 31$ days. Although we cannot definitively explain the discrepancy between spectral constraints and SN $1980 \mathrm{~K}$ model comparisons, it is possible that where Dwek $(1983,1985)$ considers a single, very thin shell of CSM material, SN 2007it may have a much more extended shell or multiple shells, creating a much later and/or longer plateau.

The IR fluxes on day 561 are very different from day 351, most importantly the fluxes in the 3.6, 5.8, and $8.0 \mu \mathrm{m}$ channels have doubled in intensity. This may indicate that new dust grains have formed. This is likely since this is the same time period in which we see a dimming in the visible light curve (between days 339 and 540). The combination of the elevated mid-IR flux at the same time as the corresponding dimming in the visible can be explained if dust grains are absorbing the high-energy photons and re-emitting them in the IR. This has been seen in other dust producing CCSNe such as SN 1987A (Suntzeff et al. 1991) and
SN 2003gd (Sugerman et al. 2006). Modified blackbody fits to this epoch indicate a blackbody luminosity of $4.5 \times 10^{38} \mathrm{erg} \mathrm{s}^{-1}$, twice the blackbody luminosity at day 351 . This is consistent with the optical drop of $0.5 \mathrm{mag}$ between the two epochs which causes the early time luminosity to be 1.58 times brighter, or almost twice the brightness of the luminosity on day 540. Within the uncertainties, the drop in optical luminosity is equal to the increase in IR luminosity.

Days 718 and 922 were both observed by Spitzer in warm mode, so only the 3.6 and $4.5 \mu \mathrm{m}$ bands were available. Both epochs show significant IR luminosity, likely due to the newly formed ejecta dust. In the day 922 data, the $4.5 \mu \mathrm{m}$ flux appears to be stronger than expected, and in fact the ratio between 3.6 and 4.5 is much smaller than other epochs, only 0.27 as opposed to 0.6 on day 718 . If we assume the ratio to be constant in the day 922 epoch, we would expect a flux of $\sim 66 \mu \mathrm{Jy}$, but instead see a flux of $143 \mu \mathrm{Jy}$, indicating that there is possibly an excess in the $4.5 \mu \mathrm{m}$ channel. This is also seen by B. E. K. Sugerman et al. (2011, in preparation) for SN 2004et. It is likely we are still seeing $\mathrm{CO}$ emission that was visible on day 351, which was hidden by the increased emission from the new dust on day 561 , and became visible again in the later epochs as the grains cooled.

\section{RADIATIVE TRANSFER MODELING}

In order to quantify the amount and composition of the dust in SN 2007it we used our three-dimensional Monte Carlo radiative transfer code MOCASSIN (Ercolano et al. 2005, and references therein). We have chosen to model three different geometries in which the dust is distributed either uniformly within a spherical shell surrounding the $\mathrm{SN}$, with the addition of clumps scattered throughout the shell, or in a torus at some inclination around the SN. These will be known as "smooth," "clumpy," and "torus" models, respectively. Relying on previous modeling done by Sugerman et al. (2006), Meikle et al. (2007), and Kotak et al. (2009), we have used a standard Mathis, Rumpi, \& Nordsieck grain size distribution of $a^{-3.5}$ between 0.005 and $0.05 \mu \mathrm{m}$ (Mathis et al. 1977) for all three scenarios. Tables 6 and 7 and Figure 7 show the model results.

As we assumed for SN 2007od (Andrews et al. 2010) in these models, the dust and luminosity for the source was located between inner radius $R_{\text {in }}$ and outer radius $R_{\text {out }}$ of a spherically expanding shell, with the luminosity being proportional to the density at each location. For day 351, we have chosen to 
concentrate the luminosity in a point source rather than spread throughout the shell, as it is likely this emission is the result of flash-heated pre-existing CSM. This does create a better fit to the IR data points. The smooth model assumes the density of dust in the shell was inversely proportional to the square of the radius. For the clumpy model, the photons originate in the inhomogeneous interclump medium, where the clumps are considered to be optically thick and spherical. For the torus models, densities are specified for the inner and outer walls, with the dust distribution falling off linearly between the two radii (Ercolano et al. 2007).

For each model we used a combination of amorphous carbon (AC) and silicate grains, using the optical constants of Hanner (1988) and Draine \& Lee (1984), respectively. For all epochs, the best fit occurred with a carbon-rich combination of $75 \% \mathrm{AC}$ and $25 \%$ silicates, as was also the case in SN 2007od (Andrews et al. 2010). For day 351 no attempt was made to fit the $4.5 \mu \mathrm{m}$ point, since it was likely due mainly to $\mathrm{CO}$ emission, the same is true for day 922 when CO may also be responsible for the elevated $4.5 \mu \mathrm{m}$ flux.

Luminosity, ejecta temperature, inner and outer radii, and dust masses were all inputs for each model. Initial estimates for each parameter were accomplished using blackbody fits to the optical and IR data. This yielded dust temperatures $\left(T_{d}\right)$ of roughly $500 \mathrm{~K}$ for the first epoch, and $700 \mathrm{~K}$ for day 561,590 $\mathrm{K}$ for day 718 , and $480 \mathrm{~K}$ for day 944 . These temperatures are consistent with typical warm dust temperatures. For our first epoch, we used an $R_{\text {in }}$ of $3.2 \times 10^{16} \mathrm{~cm}$, which roughly corresponds to the evaporation radius of the initial flash of the SN. All other epochs used an $R_{\text {in }}$ of $7 \times 10^{15} \mathrm{~cm}$ which created the best fits. We also kept the temperature of the ejecta $\left(T_{\text {ej }}\right)$ at $5700 \mathrm{~K}$ for all epochs, since this is a reasonable ejecta temperature and does an adequate job fitting the first epoch of optical points. The combination of high $\mathrm{H} \alpha$ flux and [OI] emission lines in the $R$ band relative to the continuum, and at later times additional flux due to the light echo, has made it unlikely that the visible photometry is consistent with any reasonable blackbody temperature. Keeping those two values constant, we then varied the luminosity, outer radii, and dust masses to get the most accurate fits. For all epochs, we found that the clumpy distribution predicted on average slightly higher dust masses than the smooth and torus models.

We estimate a dust mass for our first epoch of $5 \times 10^{-4} M_{\odot}$ for the smooth model, $7.3 \times 10^{-4} M_{\odot}$ for clumpy, and $1.6 \times$ $10^{-4} M_{\odot}$ for the torus. The remaining epochs yielded smooth dust masses of $7.0 \times 10^{-5} M_{\odot}$ for day $561,8.0 \times 10^{-5} M_{\odot}$ for day 718 , and $4.6 \times 10^{-5} M_{\odot}$ for day 922 . The larger dust mass from the first epoch might be explained by the flash heating of pre-existing CSM dust in an area much further away and roughly $200 \mathrm{~K}$ cooler than following epochs. At this first epoch $R_{\text {in }}=3.2$ $\times 10^{16} \mathrm{~cm}$, which is larger than the $R_{\text {out }}$ of the remaining epochs. This pre-existing dust will cool, as described in Section 5 above, and fade after its initial heating by the SN flash so that by day 561 the IR SED is dominated by newly formed ejecta dust. After day $561, R_{\text {in }}=7 \times 10^{15}$ and $R_{\text {out }}=2 \times 10^{16}$, which likely places the dust at this time in the CDS interior to the point of CSM interaction. These results also indicate that the amount of new dust created is smaller than the amount in the CSM.

On day 351, the MOCASSIN models estimate that $\tau_{v}=0.08$. In order to independently estimate the optical depth, we used the methods of Fox et al. (2009) which uses total peak energy and $\operatorname{IR} \operatorname{energy}\left(\tau \sim \frac{E_{\mathrm{IR}}}{E_{\mathrm{IR}}+E}\right)$. The peak luminosity of SN 2007it was $\sim 10^{9} L_{\odot}$, which would indicate $E \sim 10^{49}$ erg using the estimates of SN 2005ip contained in Fox et al. (2009). The IR energy for the first 351 days is then $1 \times 10^{48} \mathrm{erg}$, using an $L_{\mathrm{bb}}$ of $8.8 \times$ $10^{6} L_{\odot}$. This yields a $\tau=0.09$, consistent with our MOCASSIN fits. Between days 351 and 561, we also find a significant increase in $\tau_{v}$, which jumps from 0.08 to 1.27 as the new dust forms. This is largely due to the order of magnitude decrease in $R_{\text {out }}$ between the two epochs, requiring similar amounts of dust in a much smaller volume. Although the optical light curve suggests only an $A_{V}=0.5$, considerations of geometry, such as the newly formed dust existing in a torus around the $\mathrm{SN}$ for which we are seeing inclined at an angle away from edge-on, could explain the higher amount estimated from MOCCASIN which would provide the total optical depth summed over the whole system, not just along our line of sight. This scenario could also explain the lack of increased attenuation in the [O I] and $\mathrm{H} \alpha$ spectral lines between days 339 and 540 when we see the other dust formation signatures, mainly the simultaneous increase in optical extinction and IR luminosity. Therefore, the total mass of new dust formed in SN 2007it appears to be $\sim 1.0 \times$ $10^{-4} M_{\odot}$, which although consistent with dust masses of other CCSNe, is still considerably smaller than the amount needed to account for the dust seen at high- $z$ (Morgan \& Edmunds 2003).

\section{SUMMARY}

This paper presents the results of a comprehensive study of SN 2007it for almost three years post-explosion. SN 2007it is a Type IIP supernova, most similar to SN 2004et, that shows some interesting characteristics and has produced $\sim 1.0 \times 10^{-4} M_{\odot}$ of dust in its ejecta, likely in the CDS created from CSM interaction. High late-time luminosities indicate that $M_{\mathrm{Ni}}=$ $0.09 M_{\odot}$ was synthesized in the explosion, greater than seen in other SNe such as 1987A and 2004et. The [Ca II]/[O I] ratios post-plateau are also much smaller than normally seen in a Type IIP, 0.70 prior to dust formation and 0.50 after. These two factors, a higher than normal Ni mass and a small [Ca II]/[O I], along with the expansion velocity of $\mathrm{H} \alpha$ at early times and of [O I] at later times suggest that SN 2007it may have had a progenitor mass $\geqslant 20 M_{\odot}$, which is quite large for a Type IIP SN. The late-time light curve and color evolution also suggest that a light echo exists around SN 2007it which will be discussed in detail in an upcoming paper. Estimates of oxygen mass suggest that $1.1-1.4 M_{\odot}$ may be present, which is consistent with the Type II SNe 2004et and 1987A. The oxygen lines also show strange behavior not previously seen in a Type II SNe. The postplateau spectra show a blueshifted component at $-1300 \mathrm{~km} \mathrm{~s}^{-1}$ to both the 6300 and $6364 \AA$ lines, with no corresponding peak at $+1300 \mathrm{~km} \mathrm{~s}^{-1}$. This is likely due to emission from a torus of oxygen-rich material surrounding the $\mathrm{SN}$ or an asymmetric blob of $\mathrm{O}$ material ejected on the forward side of the $\mathrm{SN}$ during the explosion.

Modeling done on SN 2007it using our MOCCASIN radiative transfer code indicates that there was pre-existing dust surrounding the SN prior to explosion, and that up to $\sim 1.0 \times 10^{-4} M_{\odot}$ formed in the ejecta after day 351 . Up to $1 M_{\odot}$ of dust would need to be produced per SN to account for the dust amounts seen at high $z$ according to the work of Todini \& Ferrara (2001) and Nozawa et al. (2003). Recently, however, Cherchneff \& Dwek (2010) have suggested that these models may overestimate these masses, which would be consistent with the values seen in nearby SNe. Even if the amount of dust produced in SN 2007it may not be large enough to account for the dust budget of early galaxies (Kozasa et al. 1989; Todini \& Ferrara 2001), it is on par with other SNe such as 2007od (Andrews et al. 2010), 
2004et (Kotak et al. 2009), and SN 2006jc (Mattila et al. 2008). Although the sample is still small, this ever-growing body of evidence seems to indicate that low-mass CCSNe such as the ones seen today are likely not major contributors to dust in the early universe. In fact, Sloan et al. (2009) and Valiante et al. (2009) have both suggested that AGB stars can be more significant contributors to dust formation in early galaxies than once thought and Wesson et al. (2010) found that SN 2008 S was likely a massive AGB star, not a supernova at all, which produced a significant amount of dust. SNe may then only play a small part in the dust formation in early galaxies, but more long-term and comprehensive observations of $\mathrm{SNe}$ are needed to definitely answer the role of SNe as dust produces in high- $z$ galaxies.

We thank the anonymous referee for the valuable suggestions that have improved this paper. We would also like to thank Nidia Morell and the rest of the Carnegie Supernova Project for allowing us access to the early time spectroscopic data, as well as Parvis Ghavamian and Nathan Smith for helpful comments. This work has been supported by NSF grant AST-0707691 and NASA GSRP grant NNX08AV36H. This work was supported by Spitzer Space Telescope RSA 1415602 and RSA 1346842, issued by JPL/Caltech. A portion of this data was obtained at the Gemini Observatory, which is operated by the Association of Universities for Research in Astronomy (AURA) under a cooperative agreement with the NSF on behalf of the Gemini partnership. The standard data acquisition has been supported by NSF grants AST-0503871 and AST-0803158 to A. U. Landolt.

\section{REFERENCES}

Andrews, J. E., et al. 2010, ApJ, 715, 541

Barbon, R., Benetti, S., Rosino, L., Cappellaro, E., \& Turatto, M. 1990, A\&A, 237, 79

Bertoldi, F., Carilli, C. L., Cox, P., Fan, X., Strauss, M. A., Beelen, A., Omont, A., \& Zylka, R. 2003, A\&A, 406, L55

Blondin, S., Prieto, J. L., Patat, F., Challis, P., Hicken, M., Kirshner, R. P., Matheson, T., \& Modjaz, M. 2009, ApJ, 693, 207

Cherchneff, I., \& Dwek, E. 2010, ApJ, 713, 1

Chevalier, R. A., Fransson, C., \& Nymark, T. K. 2006, ApJ, 641, 1029

Chugai, N. N. 1988a, Ap\&SS, 146, 375

Chugai, N. N. 1988b, Sov. Astron. Lett., 14, 334

Contreras, C., Morrell, N., Gonzalez, S., \& Lee, K. 2007, CBET, 1068, 1

Crockett, R. M., Smartt, S. J., Pastorello, A., Eldridge, J. J., Stephens, A. W., Maund, J. R., \& Mattila, S. 2011, MNRAS, 410, 2767

Dessart, L., Livne, E., \& Waldman, R. 2010, MNRAS, 408, 87

Draine, B. T., \& Lee, H. M. 1984, ApJ, 285, 89

Dwek, E. 1983, ApJ, 274, 175

Dwek, E. 1985, ApJ, 297, 719

Dwek, E., \& Cherchneff, I. 2011, ApJ, 727, 63

Dwek, E., Galliano, F., \& Jones, A. P. 2007, ApJ, 662, 927

Eldridge, J. J., \& Tout, C. A. 2004, MNRAS, 353, 87

Elmhamdi, A., Danziger, I. J., Cappellaro, E., Della Valle, M., Gouiffes, C., Phillips, M. M., \& Turatto, M. 2004, A\&A, 426, 963

Elmhamdi, A., et al. 2003, MNRAS, 338, 939

Ercolano, B., Barlow, M. J., \& Storey, P. J. 2005, MNRAS, 362, 1038

Ercolano, B., Barlow, M. J., \& Sugerman, B. E. K. 2007, MNRAS, 375, 753

Evans, R. O., Nitschki, D., \& Quirk, S. 2007, CBET, 1065, 1

Fox, O. D., Chevalier, R. A., Dwek, E., Skrutskie, M. F., Sugerman, B. E. K., \& Leisenring, J. M. 2010, ApJ, 725, 1768

Fox, O., et al. 2009, ApJ, 691, 650

Fransson, C., \& Chevalier, R. A. 1987, ApJ, 322, L15

Fransson, C., \& Chevalier, R. A. 1989, ApJ, 343, 323

Fukugita, M., Ichikawa, T., Gunn, J. E., Doi, M., Shimasaku, K., \& Schneider, D. P. 1996, AJ, 111, 1748

Grassberg, E. K., Imshennik, V. S., \& Nadyozhin, D. K. 1971, Ap\&SS, 10, 28

Hamuy, M. 2003, ApJ, 582, 905
Hanner, M. 1988, in NASA Conf. Pub. 3004, Infrared Observations of Comets Halley and Wilson and Properties of the Grains (Washington, DC: NASA), 22

Heger, A., Fryer, C. L., Woosley, S. E., Langer, N., \& Hartmann, D. H. 2003, ApJ, 591,288

Hendry, M. A., et al. 2005, MNRAS, 359, 906

Holtzman, J. A., Burrows, C. J., Casertano, S., Hester, J. J., Trauger, J. T., Watson, A. M., \& Worthey, G. 1995, PASP, 107, 1065

Itagaki, K., et al. 2007, IAU Circ., 8874, 3

Jeffery, D. J., \& Branch, D. 1990, in Supernovae, Jerusalem Winter School for Theoretical Physics, ed. J. C. Wheeler, T. Piran, \& S. Weinberg (Singapore: World Scientific), 149

Kasen, D., \& Woosley, S. E. 2009, ApJ, 703, 2205

Kawabata, K. S., Tanaka, M., Maeda, K., Hattori, T., Nomoto, K., Tominaga, N., \& Yamanaka, M. 2009, ApJ, 697, 747

Kiewe, M., et al. 2010, arXiv:1010.2689

Kotak, R., Meikle, P., van Dyk, S. D., Höflich, P. A., \& Mattila, S. 2005, ApJ, 628, L123

Kotak, R., et al. 2006, ApJ, 651, L117

Kotak, R., et al. 2009, ApJ, 704, 306

Kozasa, T., Hasegawa, H., \& Nomoto, K. 1989, ApJ, 344, 325

Kozasa, T., Nozawa, T., Tominaga, N., Umeda, H., Maeda, K., \& Nomoto, K. 2009, in ASP Conf. Ser. 414, ed. T. Henning, E. Grün, \& J. Steinacker (San Francisco, CA: ASP), 43

Leonard, D. C., Filippenko, A. V., Barth, A. J., \& Matheson, T. 2000, ApJ, 536, 239

Lucy, L. B., Danziger, I. J., Gouiffes, C., \& Bouchet, P. 1989, in Lecture Notes in Physics, Vol. 350, IAU Colloq. 120, Structure and Dynamics of the Interstellar Medium, ed. G. Tenorio-Tagle, M. Moles, \& J. Melnick (Berlin: Springer), 164

Maeda, K., Taubenberger, S., Sollerman, J., Mazzali, P. A., Leloudas, G., Nomoto, K., \& Motohara, K. 2010, ApJ, 708, 1703

Maeda, K., et al. 2007, ApJ, 658, L5

Maguire, K., et al. 2010, MNRAS, 284

Mathis, J. S., Rumpl, W., \& Nordsieck, K. H. 1977, ApJ, 217, 425

Mattila, S., et al. 2008, MNRAS, 389, 141

Meikle, W. P. S., et al. 2007, ApJ, 665, 608

Michałowski, M. J., Watson, D., \& Hjorth, J. 2010, ApJ, 712, 942

Milisavljevic, D., Fesen, R. A., Gerardy, C. L., Kirshner, R. P., \& Challis, P. 2010, ApJ, 709, 1343

Miller, A. A., Smith, N., Li, W., Bloom, J. S., Chornock, R., Filippenko, A. V., \& Prochaska, J. X. 2010, AJ, 139, 2218

Misra, K., Pooley, D., Chandra, P., Bhattacharya, D., Ray, A. K., Sagar, R., \& Lewin, W. H. G. 2007, MNRAS, 381, 280

Modjaz, M., Kirshner, R. P., Blondin, S., Challis, P., \& Matheson, T. 2008, ApJ, 687, L9

Morgan, H. L., \& Edmunds, M. G. 2003, MNRAS, 343, 427

Nozawa, T., Kozasa, T., Umeda, H., Maeda, K., \& Nomoto, K. 2003, ApJ, 598, 785

Pastorello, A., et al. 2005, MNRAS, 360, 950

Pastorello, A., et al. 2009, MNRAS, 394, 2266

Patat, F. 2005, MNRAS, 357, 1161

Pojmanski, G. 2007, IAU Circ., 8875, 1

Pronik, V. I., Chuvaev, K. K., \& Chugai, N. N. 1976, SvA, 20, 666

Roche, P. F., Aitken, D. K., \& Smith, C. H. 1991, MNRAS, 252, 39P

Sahu, D. K., Anupama, G. C., Srividya, S., \& Muneer, S. 2006, MNRAS, 372 , 1315

Sirianni, M., et al. 2005, PASP, 117, 1049

Sloan, G. C., et al. 2009, Science, 323, 353

Smartt, S. J., Eldridge, J. J., Crockett, R. M., \& Maund, J. R. 2009, MNRAS, 395, 1409

Smith, N., Foley, R. J., \& Filippenko, A. V. 2008, ApJ, 680, 568

Smith, N., et al. 2009, ApJ, 695, 1334

Sugerman, B. E. K. 2003, AJ, 126, 1939

Sugerman, B. E. K. 2005, ApJ, 632, L17

Sugerman, B. E. K., et al. 2006, Science, 313, 196

Suntzeff, N. B., Phillips, M. M., Depoy, D. L., Elias, J. H., \& Walker, A. R. 1991, AJ, 102, 1118

Szalai, T., Vinkó, J., Balog, Z., Gáspár, A., Block, M., \& Kiss, L. L. 2011, A\&A, 527,61

Taubenberger, S., et al. 2009, MNRAS, 397, 677

Todini, P., \& Ferrara, A. 2001, MNRAS, 325, 726

Tully, R. B. (ed.) 1988, Nearby Galaxies Catalog (Cambridge: Cambridge Univ. Press)

Tully, R. B., Shaya, E. J., Karachentsev, I. D., Courtois, H. M., Kocevski, D. D., Rizzi, L., \& Peel, A. 2008, ApJ, 676, 184 
Turatto, M., Cappellaro, E., Benetti, S., \& Danziger, I. J. 1993, MNRAS, 265, 471

Uomoto, A. 1986, ApJ, 310, L35

Valiante, R., Schneider, R., Bianchi, S., \& Andersen, A. C. 2009, MNRAS, 397, 1661

Vinkó, J., et al. 2006, MNRAS, 369, 1780

Weaver, T. A., \& Woosley, S. E. 1980, in Annals of the New York Academy of Sciences, Vol. 336, Ninth Texas Symposium on Relativistic Astrophysics, ed. J. Ehlers, J. J. Perry, \& M. Walker (New York, NY: New York Academy of Sciences), 335
Welch, D. L., Clayton, G. C., Campbell, A., Barlow, M. J., Sugerman, B. E. K. Meixner, M., \& Bank, S. H. R. 2007, ApJ, 669, 525

Wesson, R., et al. 2010, MNRAS, 403, 474

Winkler, P. F., \& Kirshner, R. P. 1985, ApJ, 299, 981

Wooden, D. H., Rank, D. M., Bregman, J. D., Witteborn, F. C., Tielens, A. G. G. M., Cohen, M., Pinto, P. A., \& Axelrod, T. S. 1993, ApJS, 88, 477

Woosley, S. E., \& Weaver, T. A. 1986, ARA\&A, 24, 205

Zhang, T., Wang, X., Li, W., Zhou, X., Ma, J., Jiang, Z., \& Chen, J. 2006, AJ, 131,2245 